\section{Variations in chest compression time, ventilation time and rescuers' heart rate during conventional cardiopulmonary resuscitation in trained male rescuers}

\author{
Jun Young Hong, Je Hyeok Oh \\ Department of Emergency Medicine, Chung-Ang University College of Medicine, Seoul, Korea
}

Objective This study was conducted to determine why rescuers could maintain adequate chest compression depth for longer periods during conventional cardiopulmonary resuscitation (CPR).

Methods Various CPR parameters, including average compression depth (ACD), average compression rate, average ventilation time (AVT), and rescuers' heart rates were recorded in real-time when 20 health care providers performed 10 minutes of conventional CPR during a simulation experiment.

Results The ACD was maintained above $50 \mathrm{~mm}$ and was not significantly different during 19 consecutive CPR cycles. The average compression rate increased from 114.9 $\pm 10.0 / \mathrm{min}$ (2nd cycle) to $120.1 \pm 13.8 / \mathrm{min}$ (18th cycle) $(P=0.007)$, and the AVT increased from $8.7 \pm 1.5$ seconds (3rd cycle) to $10.1 \pm 2.6$ seconds ( 18 th cycle) $(P=0.002)$. The rescuers' heart rates also increased gradually for 10 minutes; however, they increased rapidly and were highest during the ventilation phase. Their heart rates then decreased and were lowest during the early chest compression phases of each CPR cycle. Decreases in heart rates were significant in all CPR cycles (average decrease: $14.5 \pm 4.5$ beats $/ \mathrm{min}, \mathrm{P}<0.001$ ).

Conclusion The ACD was maintained adequately during 10 minutes of conventional CPR. However, the AVT increased significantly during the 10-minute period. The rescuers' heart rates increased and decreased throughout all CPR cycles. These results showed that the ventilation phase might play a role as a resting period and be a reason for the maintenance of adequate chest compression depth for prolonged periods during conventional CPR.

Keywords Cardiopulmonary resuscitation; Heart rate; Ventilation; Fatigue

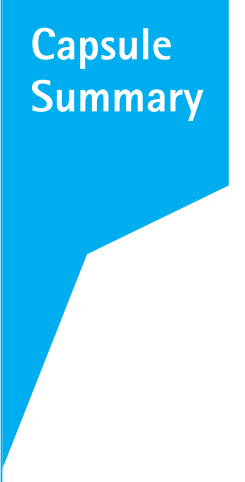

What is already known

Although the quality of cardiopulmonary resuscitation (CPR) decreases with time, the speed of decay is slower in conventional CPR than in chest-compression-only CPR. It is believed that the ventilation phase during conventional CPR may function as a resting period for rescuers; however, the evidence supporting this belief has not been reported.

What is new in the current study

The average ventilation time increased significantly during 10 minutes, and the rescuers' heart rates increased and decreased throughout all CPR cycles. These results showed that the ventilation phase might play a role as a resting period and be a reason for the maintenance of adequate chest compression depth for prolonged durations during conventional CPR.
elSSN: 2383-4625

Received: 9 October 2017

Revised: 1 November 2017

Accepted: 3 November 2017

Correspondence to: Je Hyeok Oh Department of Emergency Medicine, Chung-Ang University College of Medicine, 84 Heukseok-ro, Dongjak-gu, Seoul 06974, Korea

E-mail: jehyeokoh@cau.ac.kr ORCID

http://orcid.org/0000-0002-5211-3838

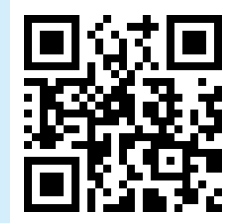

How to cite this article:

Hong JY, Oh JH. Variations in chest compression time, ventilation time and rescuers' heart rate during conventional cardiopulmonary resuscitation in trained male rescuers. Clin Exp Emerg Med 2019;6(1):31-35.

This is an Open Access article distributed under the terms of the Creative Commons Attribution Non-Commercial License (http:// creativecommons.org/licenses/by-nc/4.0/). 


\section{INTRODUCTION}

Maintaining adequate chest compression depth (CCD) during cardiopulmonary resuscitation (CPR) is closely related to patient survival. ${ }^{1}$ The 2015 International Consensus on Cardiopulmonary Resuscitation and Emergency Cardiovascular Care Science with Treatment Recommendations advise rotating providers performing chest compressions every 2 minutes because the CCD decreases after 90 seconds of continuous chest compressions (CCCs). ${ }^{2,3}$ Although the quality of CPR also decreases during conventional CPR, the speed of decay is faster in CCC than in conventional $\mathrm{CPR}^{4,5} \mathrm{It}$ is believed that the ventilation phase during conventional CPR may function as a resting period for rescuers; however, the evidence supporting this belief is lacking. This study was conducted to determine the reasons why rescuers could maintain adequate $C C D$ for prolonged durations during conventional CPR.

\section{METHODS}

\section{Study protocol}

This study was a pilot study using an adult cardiac arrest simulation. The study protocol was approved by the institutional review board of the Chung-Ang University Hospital (C2015107[1565]). After providing their written informed consent, 20 health care providers (all certified American Heart Association Basic Life Support providers; one attending physician, two resident physicians, and 17 intern physicians) each performed 10 minutes of conventional CPR with a 30:2 chest compression to ventilation ratio using an adult cardiac arrest simulation on the floor. A Little Anne manikin (Laerdal Medical, Stavanger, Norway) was used as a simulated cardiac arrest patient. Mouth-to-mouth ventilation was performed with a face shield using the head-tilt/chin-lift maneuver. The performance of CPR was recorded using a CPRmeter (Laerdal Medical). The feedback screen was covered during the experiments. The rescuers' real-time heart rates were recorded using the POLAR H7 heart rate sensor (Polar Electro Oy, Kempele, Finland), and the data were collected at 0.5-second intervals using the Heart Graph application (Smoky Cat Software Ltd., Warwick, UK).

\section{Outcome variables}

We selected several CPR parameters, including the average chest compression depth (ACD), average chest compression rate (ACR), average chest compression time in each CPR cycle (ACT), and average ventilation time in each CPR cycle (AVT), for the statistical analyses. All parameters were calculated using the average of each CPR cycle. In addition, we recorded the rescuers' heart rates in real-time for 10 minutes.

\section{Statistical analyses}

All analyses were performed using the IBM SPSS Statistics ver. 23.0 (IBM Corp., Armonk, NY, USA). The variables were presented as mean \pm standard deviation. The Friedman test was used to determine whether the averaged CPR parameters in each CPR cycle changed significantly during the 10-minute period. In addition, the Wilcoxon signed-rank test was used to compare the variables between two different CPR cycles and to determine whether the rescuers' heart rates decreased significantly in each CPR cycle. Pvalues $<0.05$ were considered statistically significant.

\section{RESULTS}

Twenty male medical doctors participated in this study. Their mean age was $27.6 \pm 3.7$ years. Their mean body weight was $73.9 \pm 10.7 \mathrm{~kg}$, and their mean height was $177.2 \pm 5.3 \mathrm{~cm}$ (average body mass index $=24.6 \pm 3.4 \mathrm{~kg} / \mathrm{m}^{2}$ ). The ACD and ACR for 10 minutes were $54.2 \pm 4.4 \mathrm{~mm}$ and $117.6 \pm 13.4$ compressions/min, respectively. The rescuers performed $23.9 \pm 2.8 \mathrm{CPR}$ cycles in 10 minutes. The ACT and AVT in all CPR cycles were $15.6 \pm 1.8$ and $9.4 \pm 1.7$ seconds, respectively. The chest compression fraction in 10 minutes was $63.7 \pm 4.3 \%$.

\section{Changes in the CPR parameters}

The ACD was maintained above $50 \mathrm{~mm}$ and was not significantly different during the 19 consecutive CPR cycles $(P=0.597)$ (Fig. 1). However, the ACR and AVT were significantly different $(P<0.001)$ (Figs. 2, 3). The ACR increased from $114.9 \pm 10.0 / \mathrm{min}$ (2nd cycle: minimum value) to $120.1 \pm 13.8 /$ min (18th cycle: maximum value) $(P=0.007)$ (Fig. 2), and the AVT increased from $8.7 \pm 1.5 \mathrm{sec}-$ onds (3rd cycle: minimum value) to $10.1 \pm 2.6$ seconds (18th cy-

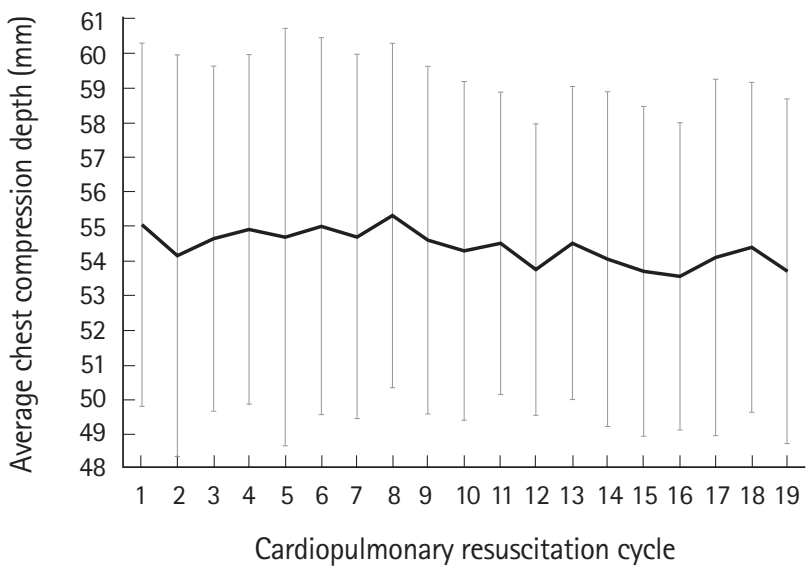

Fig. 1. Changes in the average chest compression depths according to the cardiopulmonary resuscitation cycles. The lines display the means, and the error bars indicate the standard deviations. 


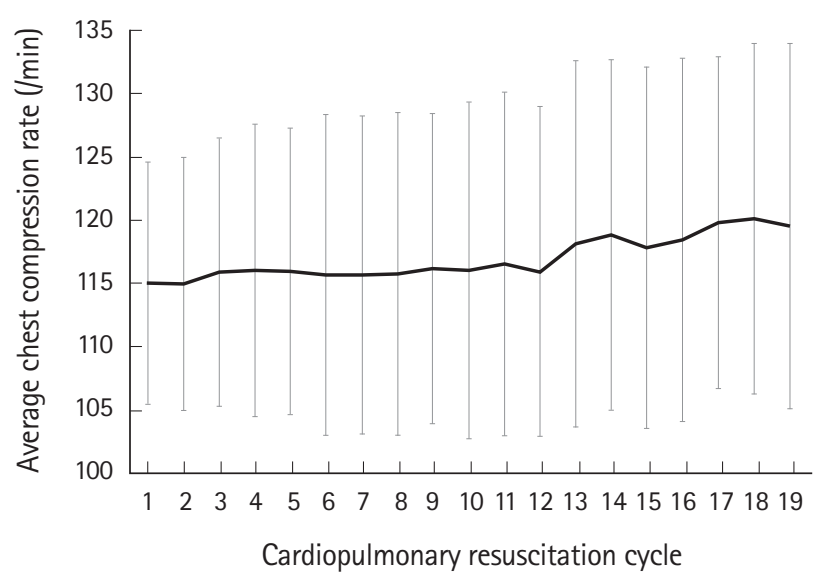

Fig. 2. Changes in the average chest compression rates according to the cardiopulmonary resuscitation cycles. The lines display the means, and the error bars indicate the standard deviations.

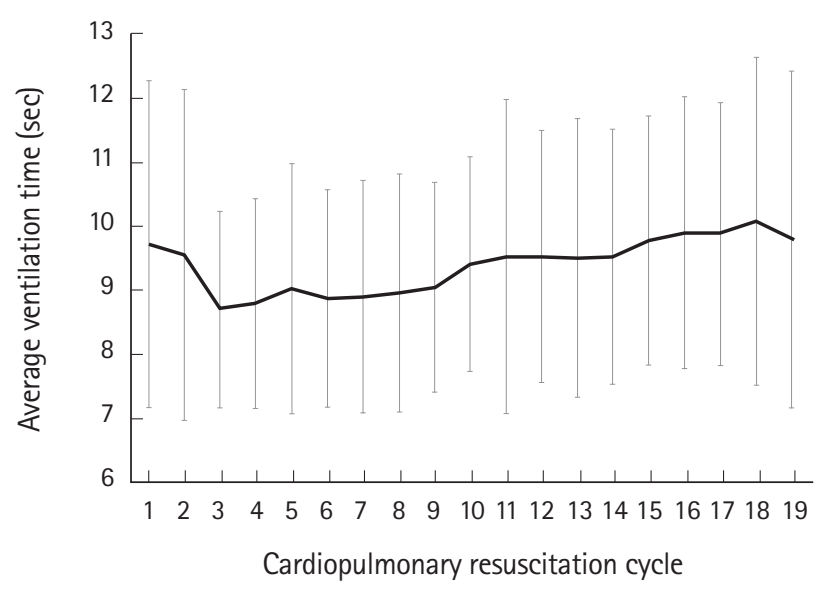

Fig. 3. Changes in the average ventilation times according to the cardiopulmonary resuscitation cycles. The lines display the means, and the error bars indicate the standard deviations.

cle: maximum value) $(P=0.002)$ (Fig. 3). The ACT was also significantly different during the 19 consecutive CPR cycles $(P=0.002)$ (Fig. 4). However, the ACT showed a decreasing trend, unlike the $\mathrm{ACR}$ and $\mathrm{AVT}$.

\section{Changes in the rescuers' heart rates}

The rescuers' heart rates increased gradually for 10 minutes (Fig. 5). However, they increased rapidly and were highest during the ventilation phase. Their heart rates then decreased and were lowest during the early chest compression phases of each CPR cycle. These changes were consistent during the 19 consecutive CPR cycles. The heart rates decreased significantly in all CPR cycles (average decrease: $14.5 \pm 4.5$ beats/min [11.7 $\pm 3.7 \%$ decrease from the preceding peak values], $\mathrm{P}<0.001$ ).

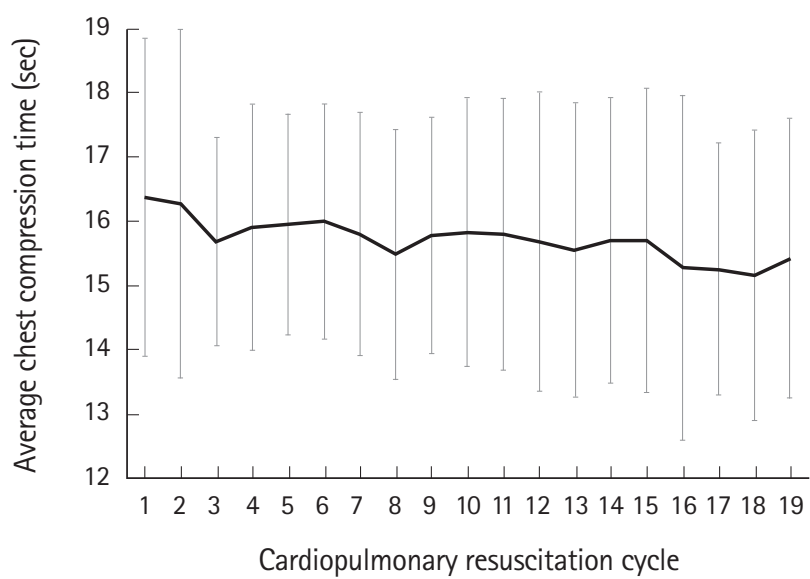

Fig. 4. Changes in the average chest compression times according to the cardiopulmonary resuscitation cycles. The lines display the means, and the error bars indicate the standard deviations.

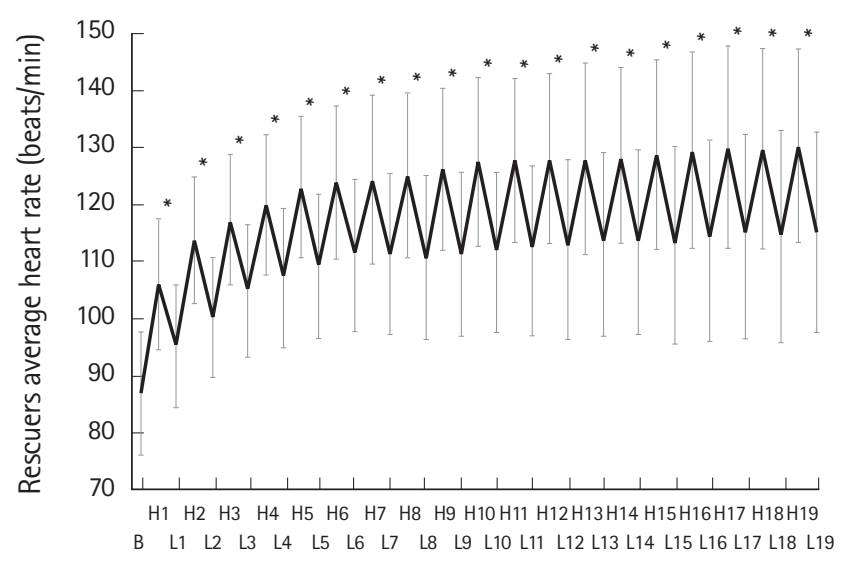

Highest and lowest heart rates in each cycle of CPR

Fig. 5. Highest and lowest rescuers' heart rates during each cardiopulmonary resuscitation (CPR) cycle. The lines display the means, and the error bars indicate the standard deviations. CPR, cardiopulmonary resuscitation; $B$, baseline heart rate; $H$, highest heart rate in each $C P R$ cycle; $\mathrm{L}$, lowest heart rate in each $\mathrm{CPR}$ cycle. ${ }^{*} \mathrm{P}<0.001$ between the highest and lowest heart rates in each CPR cycle.

\section{DISCUSSION}

To the best of our knowledge, this was the first study to evaluate changes in various CPR parameters including ACT and AVT and to measure rescuers' heart rates in real-time during prolonged conventional CPR. Although some results were expected, other findings were unexpected. The expected results were changes in $A C D$, $A C R$ and $A C T$. The unexpected findings were changes in AVT and rescuers' heart rates. The ACD did not change during the $10 \mathrm{~min}-$ utes of conventional CPR. Although the duration of our experiments was longer than previous studies, this result was basically the same as those of previous studies. ${ }^{4,5}$ The increasing ACR also 
confirmed the results of previous studies. ${ }^{6}$ The rescuers tended to compress the patient's thorax faster as fatigue increased over time. We can easily expect that the increase in ACR reflects the rescuers' fatigue because $A C R$ increased in proportion to time. ${ }^{6}$ The decrease in ACT was caused by increasing ACR because the number of chest compressions in each CPR cycle was fixed at 30.

Increasing AVT and changing patterns of rescuers' heart rate were unexpected results. We expected that the rescuers' heart rates would increase slowly throughout the experiment as fatigue increased. Previous studies also showed increasing patterns throughout the experiments. ${ }^{4,8}$ The difference in our study was that we measured the rescuers' heart rates in real-time, and this enabled us to record real changes in rescuers' heart rates within the CPR cycles, especially in relation to the ventilation phase. Our results showed that the rescuers' heart rates decreased significantly immediately after the ventilation phase of each CPR cycle. This result was clear evidence supporting the fact that the ventilation phase of each CPR cycle might act as a resting period. In addition, as rescuers' heart rates increased and decreased with CPR cycles, the heart rate graph showed a serrated shape (Fig. 5). Although the rescuers' heart rates decreased significantly immediately after the ventilation phase in all CPR cycles, it did not decrease to the baseline level. This result suggests that the rescuers' fatigue could not be relieved completely during the ventilation phase of each CPR cycle.

The increasing AVT was also an unexpected finding. The AVTs of the first and second CPR cycles were higher than that of the third CPR cycle. This result might be caused by the adaptation period of the experiments because the AVT increased gradually after the third CPR cycle (Fig. 3). We supposed that the rescuers tended to increase the ventilation time according to the accumulation of fatigue. In summary, it can be assumed that the CCD was maintained adequately for a long period because of the ventilation phase.

There were several limitations to the present study. First, we recorded the rescuers' heart rates as an objective index representing fatigue. However, heart rate alone is insufficient in determining the rescuers' fatigue, as previously described..$^{9}$ Other factors, such as recent exercise, working hours, caffeine consumption, rescuers' emotional states, and stress levels could affect the heart rate. Second, we recruited only male medical doctors to avoid ethical issues because we attached a heart rate sensor to the participant's anterior chest wall to monitor heart rate in realtime during the trial. Thus, this could limit the generalizability of our findings. Third, we recruited only 20 participants without calculating the number of study participants because this was a pilot study. As a result, we could not perform multiple comparisons among the variables of each CPR cycle. Further studies are needed to confirm the exact trends of the variables over time. Fourth, we collected data using simulation experiments with a mechanical model. Therefore, the results have a limited applicability to human patients. In addition, the researchers observed all experiments during data collection. Therefore, the Hawthorne effect might have occurred. Fifth, the present study did not include the data from CCC. Therefore, we did not compare the parameters between CCC and conventional CPR. Despite these limitations, we believe that our data can provide theoretical evidence for CPR performance and physiologic changes in rescuers during conventional CPR.

In summary, the ACD was maintained adequately during 10 minutes of conventional CPR. However, the AVT increased significantly during the 10-minute period. The rescuers' heart rates increased and decreased throughout all CPR cycles. These results showed that the ventilation phase might play a role as a resting period and be a reason for the maintenance of adequate CCD for a longer time during conventional CPR.

\section{CONFLICT OF INTEREST}

No potential conflict of interest relevant to this article was reported.

\section{REFERENCES}

1. Stiell IG, Brown SP, Nichol G, et al. What is the optimal chest compression depth during out-of-hospital cardiac arrest resuscitation of adult patients? Circulation 2014;130:1962-70.

2. Travers $A H$, Perkins GD, Berg RA, et al. Part 3: adult basic life support and automated external defibrillation. 2015 International Consensus on Cardiopulmonary Resuscitation and Emergency Cardiovascular Care Science with Treatment Recommendations. Circulation 2015;132(16 Suppl 1):S51-83.

3. Sugerman NT, Edelson DP, Leary M, et al. Rescuer fatigue during actual in-hospital cardiopulmonary resuscitation with audiovisual feedback: a prospective multicenter study. Resuscitation 2009;80:981-4.

4. Shin J, Hwang SY, Lee HJ, et al. Comparison of CPR quality and rescuer fatigue between standard 30:2 CPR and chest compression-only CPR: a randomized crossover manikin trial. Scand J Trauma Resusc Emerg Med 2014;22:59.

5. Hong DY, Park SO, Lee KR, Baek KJ, Shin DH. A different rescuer changing strategy between 30:2 cardiopulmonary resuscitation and hands-only cardiopulmonary resuscitation that considers rescuer factors: a randomized cross-over simulation 
study with a time-dependent analysis. Resuscitation 2012;83: 353-9.

6. Badaki-Makun 0, Nadel F, Donoghue A, et al. Chest compression quality over time in pediatric resuscitations. Pediatrics 2013;131:e797-804.

7. Lopez-Gonzalez A, Sanchez-Lopez M, Rovira-Gil E, GonzalezGarcia A, Ferrer-Lopez V, Martinez-Vizcaano V. Sex differences in the effort indicators during cardiopulmonary resuscita- tion manoeuvres on manikins. Eur J Emerg Med 2015;22:62-5.

8. Hasegawa T, Daikoku R, Saito S, Saito Y. Relationship between weight of rescuer and quality of chest compression during cardiopulmonary resuscitation. J Physiol Anthropol 2014;33: 16.

9. Mpotos N, Monsieurs K, Hachimi-Idrissi S. Effect of rescuer's sex on the quality of chest compression during cardiopulmonary resuscitation on manikins. Eur J Emerg Med 2015;22:69. 